\title{
Reevaluation of the value of prolactin in differentiating malignant from benign pituitary stalk thickening: Letter to the Editor
}

\author{
Shirui Wang ${ }^{1}$, Xiang Zhou' ${ }^{2}$ and Huijuan Zhu ${ }^{2}$ \\ ${ }^{1}$ Eight-Year Program of Clinical Medicine, Peking Union Medical College Hospital, Chinese Academy of Medical Sciences \& Peking Union \\ Medical College, Beijing, China and 2Department of Endocrinology, Key Laboratory of Endocrinology of National \\ Health Commission, Peking Union Medical College Hospital, Chinese Academy of Medical Science and Peking Union \\ Medical College, Beijing, China
}

Correspondence

should be addressed

to $\mathrm{H}$ Zhu

Email

shengxin2004@163.com

\section{Dear Editor,}

We have read with great interest the recent manuscript published in the European Journal of Endocrinology concerning the distinction between neoplastic and non-neoplastic pituitary stalk thickening (PST) (1). In a retrospective cohort including 38 patients diagnosed with PST and central diabetes insipidus (CDI), France Devuyst and colleagues observed a higher level of serum prolactin (PRL) in patients with malignant diseases (3.10× upper limit of normal (ULN), P5-P95 0.75-6.25) compared with benign diseases (1.15× ULN, P5-P95 0.35-3.02) $(P=0.001)$. The PRL for germinoma (2.79× ULN, P5-P95 0.75-4.62) was also higher than that for histiocytosis (1.15× ULN, P5-P95 0.70-1.78) $(P<0.01)$ and neuroinfundibulitis (1.00× ULN, P5-P95 0.35-2.81) $(P<0.01)$. The highest diagnostic accuracy for differentiating between neoplastic and benign PST was observed for a PRL cut-off of $1.27 \times$ ULN (sensitivity 90\%, specificity 67\%), and the area under the curve (AUC) for the Receiver Operating Characteristic Curve (ROC) was $0.852(P=0.002)$. This article is of clinical significance considering the difficulties in the differential diagnosis of PST. In this letter, based on our PST cohort in one of the largest pituitary multi-discipline centers in China, we aimed to provide more evidence in regard to the predictive value of PRL level in distinguishing malignant and benign PST lesions.

PST and CDI patients with baseline PRL values admitted to Peking Union Medical College Hospital from January 2014 to May 2017 were retrospectively included. In accordance with Devuyst's criteria, patients younger than 15 years old, and those with Rathke's cleft cyst, craniopharyngioma, or metastases were excluded. For details in PST definition and diagnostic criteria, please refer to Xiang Zhou's article (2). It remained controversial about the classification of Langerhans cell histiocytosis. For comparison, we classified ' $\mathrm{L}$ ' type histiocytosis as benign lesions as Devuyst did (1). Since the definition of PRL ULN was not reported in the Devuyst's study, we set the ULN as $25 \mu \mathrm{g} / \mathrm{L}$ for women, and $20 \mu \mathrm{g} / \mathrm{L}$ for men according to Guidelines of the Pituitary Society for the diagnosis and management of prolactinomas (3).

Forty-six patients were included in the final analysis. There were 30 patients in the benign group, and 16 patients in the malignant group. The etiologies of included patients were shown in Table 1, of which 40 patients were diagnosed pathologically, and 6 patients (5 patients with lymphocytic hypophysitis, 1 patient with IgG4-related hypophysitis) were diagnosed clinically.

The distinction in the PRL level was not observed between malignant (1.66× ULN, P5-P95 0.40-4.42) and benign group $(1.11 \times$ ULN, P5-P95 0.31-5.56) $(P=0.201)$. The best cut-off to differentiate malignant from benign lesions was $1.03 \times$ ULN (sensitivity $87.5 \%$, specificity $50.0 \%$ ), and the AUC value was 0.617 (Fig. 1). The best cut-off value reported by Devuyst, 1.27× ULN, yielded a sensitivity of $68.8 \%$ and a specificity of $53.3 \%$. We also compared the PRL level for specific etiologies and found no significant difference between germinoma $(1.36 \times \mathrm{ULN}$, P5-P95 0.39-4.57) and histiocytosis (1.34× ULN, P5-P95 0.43-3.40) $(P=0.597)$ or hypophysitis $(0.92 \times$ ULN, P5P95 0.25-6.76) ( $P=0.177)$ (Fig. 2). A logistic regression analysis adjusted for age, sex, and lesion extension

Published by Bioscientifica Ltd. 
Table 1 Etiologies of included patients $(n=46)$.

\begin{tabular}{lll}
\hline Benign disease $(n=30)$ & & Malignant disease $(n=16)$ \\
\hline Hypophysitis (11) & & Germinoma (15) \\
Lymphocytic hypophysitis (10) & Spindle cell carcinoma (1) \\
IgG4-related hypophysitis (1) & & \\
Histiocytosis (17) & \\
LCH (14) & \\
ECD (2) & \\
Mixed LCH and ECD (1) & \\
Pituitary abscess (1) & \\
Pituitary adenoma (1)
\end{tabular}

ECD, Erdheim-Chester disease; LCH, Langerhans cell histiocytosis.

beyond pituitary stalk was performed, and we found no relationship between PRL and malignant/benign etiologies (odds ratio (OR): 0.65, 95\% CI: 0.32-1.15).

Our analysis showed no difference in PRL level between malignant and benign lesions, and the prediction value of PRL in the differential diagnosis of PST seems to be limited. Tough the best cut-off $1.03 \times$ ULN could detect $87.5 \%$ malignant PST, $50 \%$ of patients with benign lesions would be misdiagnosed. And the diagnostic accuracy for a PRL value above $1.27 \times$ in our cohort was much lower than Devuyst's report.

In contrast to Devuyst's results, we found a large overlap in PRL level among patients with germinoma,

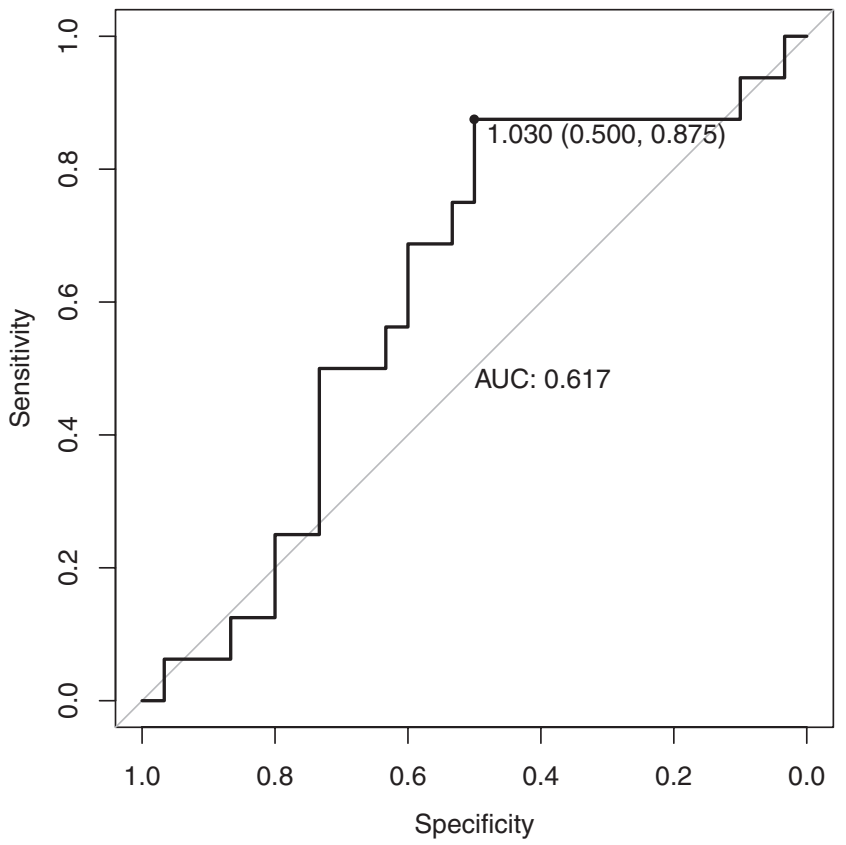

Figure 1

ROC curves of prolactin level for the distinction of malignant from benign pituitary stalk thickening. The best cut-off for prolactin was $1.03 \times$ ULN (sensitivity $87.5 \%$, specificity $50.0 \%$, AUC 0.617). hypophysitis and histiocytosis. The PRL secretion in hypophysitis patients could be decreased, normal, or elevated, and the elevation accounts for 30-48\% patients $(4,5)$. The possible mechanisms of hyperprolactinemia in hypophysitis includes stalk compression, impaired dopamine production due to inflammation, antibodies stimulating PRL secretion, and increased PRL release as a result of inflammatory infiltration of the anterior pituitary gland $(6,7)$. Therefore, using high PRL as a criterion for the diagnosis of malignant PST might misdiagnose a certain number of patients with concomitant hypophysitis and hyperprolactinemia. Future studies should be done to explore the difference between hypophysitis with and without PRL elevation to assist differential diagnosis. As for ' $\mathrm{L}$ ' type histiocytosis, we are more inclined to consider it as a malignant entity rather than a benign disease. From the perspective of pathogenesis, ' $L$ ' type histiocytosis originated from MAPK activation of myeloid precursor cells, suggesting a neoplastic origin. Moreover, the standard treatment of Langerhans cell histiocytosis, a major form of ' $\mathrm{L}$ ' type histiocytosis, was chemotherapy, which was generally applied in cancer treatment (8). In our current cohort analysis, PRL level in histiocytosis was more approximate to those in the malignant group.

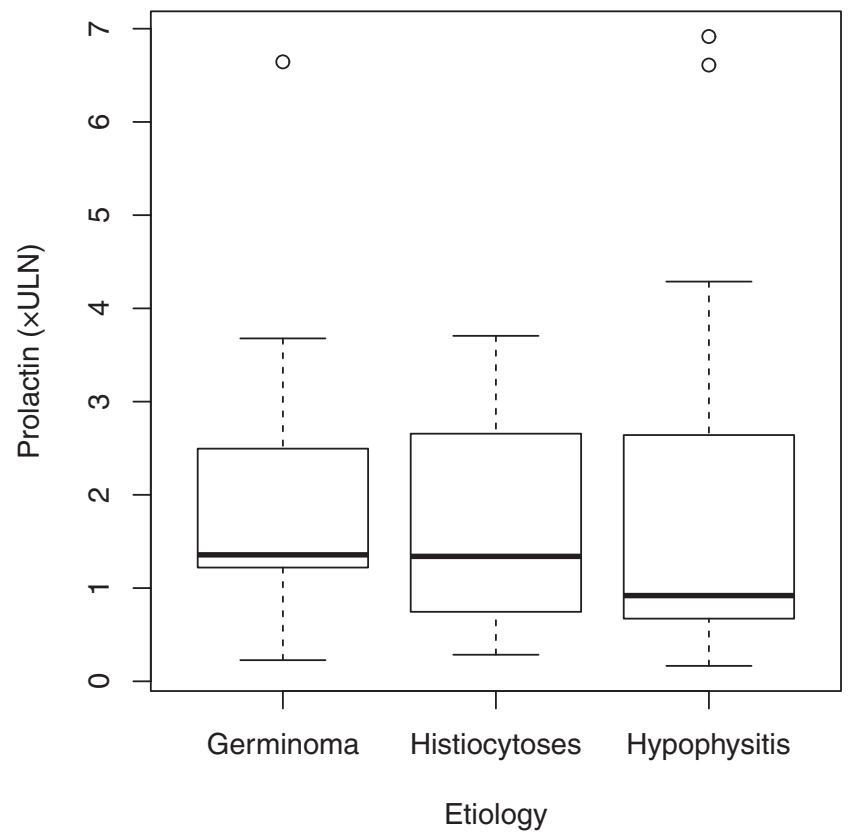

Figure 2

Serum prolactin level in patients with germinoma, histiocytosis and hypophysitis. Boxplots represent the medians, interquartile ranges and (P5-P95) intervals. 
The difference between our and Devuyst's results might partly lie in the patients' characteristics. The serum prolactin level was reported to be associated with demographic characteristics such as age and sex (9). In Devuyst's cohort, the male-female ratio was significantly higher in the malignant group ( $9 / 2$ vs $11 / 16, P=0.021)$, and the age between the malignant and benign group was comparable $(32.7 \pm 22.4$ vs $38.7 \pm 17.2$ years, $P=0.378)$. However, in our study, female patients were more than male patients not only in benign group (17/13) but also in the malignant group $(10 / 6)(P=0.702)$, and the patients in the malignant group (18.7 years, interquartile range (IQR): 16.8-24.7) was significantly younger than those in the benign group (40.9 years, IQR: $30.5-47.7)(P<0.001)$. Our logistic regression model showed that after adjusting for potential confounding factors, there was no association between PRL and PST etiologies, and a similar result was observed in a study including 158 patients with PST (10). Therefore, the different composition of the study population might result in PRL variance, and consequently the difference in diagnostic accuracy.

In summary, based on our cohort analysis, there was no significant difference in the PRL level between malignant and benign lesions. PRL lower than 1.03× ULN could partly help to rule out malignant lesions, but the large overlap among different etiologies made the diagnostic accuracy unsatisfactory. Larger studies are warranted to validate our findings, and the best cut-off point to differentiate malignant from benign PST needs to be further confirmed.

\section{Declaration of interest}

The authors declare that there is no conflict of interest that could be perceived as prejudicing the impartiality of this letter.

\section{Funding}

This research did not receive any specific grant from any funding agency in the public, commercial or not-for-profit sector.

\section{References}

1 Devuyst F, Kazakou P, Balériaux D, Alexopoulou O, Burniat A, Salenave S, Chanson P, Vorvilain B \& Maiter D. Central diabetes insipidus and pituitary stalk thickening in adults: distinction of neoplastic from non-neoplastic lesions. European Journal of Endocrinology 2020181 95-105. (https://doi.org/10.1530/EJE-20-0058)

2 Zhou X, Zhu H, Yao Y, Lian X, Feng F, Wang L, Liu S, Deng K, You H, Yang $\mathrm{H}$ et al. Etiological spectrum and pattern of change in pituitary stalk thickening: experience in 321 patients. Journal of Clinical Endocrinology and Metabolism 2019104 3419-3427. (https://doi. org/10.1210/jc.2018-02297)

3 Casanueva FF, Molitch ME, Schlechte JA, Abs R, Bonert V, Bronstein MD, Brue T, Cappabianca P, Colao A, Fahlbusch R et al. Guidelines of the pituitary society for the diagnosis and management of prolactinomas. Clinical Endocrinology 200665 265-273. (https:// doi.org/10.1111/j.1365-2265.2006.02562.x)

4 Imber BS, Lee HS, Kunwar S, Blevins LS \& Aghi MK. Hypophysitis: a single-center case series. Pituitary 201518 630-641. (https://doi. org/10.1007/s11102-014-0622-5)

5 Beressi N, Beressi JP, Cohen R \& Modigliani E. Lymphocytic hypophysitis. A review of 145 cases. Annales de Medecine Interne 1999 $150327-341$

6 De Bellis A, Bizzarro A, Pivonello R, Lombardi G \& Bellastella A. Prolactin and autoimmunity. Pituitary 20058 25-30. (https://doi. org/10.1007/s11102-005-5082-5)

7 Thodou E, Asa SL, Kontogeorgos G, Kovacs K, Horvath E \& Ezzat S. Clinical case seminar: lymphocytic hypophysitis: clinicopathological findings. Journal of Clinical Endocrinology and Metabolism 199580 2302-2311. (https://doi.org/10.1210/jcem.80.8.7629223)

8 Allen CE, Merad M \& McClain KL. Langerhans-cell histiocytosis. New England Journal of Medicine 2018379 856-868. (https://doi. org/10.1056/NEJMra1607548)

9 Roelfsema F, Pijl H, Keenan DM \& Veldhuis JD. Prolactin secretion in healthy adults is determined by gender, age and body mass index. PLoS ONE 20127 e31305. (https://doi.org/10.1371/journal.pone.0031305)

10 Lee JY, Park JE, Shim WH, Jung SC, Choi CG, Kim SJ, Kim JH \& Kim HS. Joint approach based on clinical and imaging features to distinguish non-neoplastic from neoplastic pituitary stalk lesions. PLOS ONE 2017 12 e0187989. (https://doi.org/10.1371/journal.pone.0187989)

Received 14 August 2020

Accepted 23 September 2020 